\title{
Arm rehabilitation in chronic stroke using neurologic music therapy: two contrasting case studies to inform on treatment delivery and patient suitability
}

CASE STUDY

Alexander J. Street (a), Jorg Fachnera,(b) and Wendy L. Magee (c)

(a) Cambridge Institute for Music Therapy Research, Anglia Ruskin University, Cambridge, UK; (b) Music, Health and the Brain, Anglia Ruskin University, Cambridge, UK; (c) Music Therapy Program, Temple University, Philadelphia, MA, USA

\section{Abstract}

Introduction: Therapeutic Instrumental Music Performance (TIMP) is well suited for upper limb rehabilitation following stroke. Published protocols serve to inform clinicians on intervention design and delivery. However, few case studies are available that address patient suitability, protocol modifications to support treatment adherence and suitability of home environment.

Methods: Two case studies from a small randomized controlled trial illustrate TIMP protocol modifications and considerations required for home delivery. Qualitative, quantitative and observational data report on participants' outcomes and engagement with six weeks of bi-weekly exercises. TIMP adaptations to enhance audio-motor synchronization are described.

Results: Outcomes for the less impaired participant with fewer complex health needs were significantly better after six weeks, particularly pinch grip ( 1 peg in 20 seconds to 15/120). The second participant improved on the water pouring task: 44 seconds to 13.16. 
Discussion: Severity of stroke and impairment are major factors influencing treatment outcomes. Flexibility in the TIMP protocols, such as emphasizing the underlying pulse and building the dynamic contour, aids treatment adherence and movement synchrony. It is essential to assess homes for access, sound containment and space. Outcome measures for detecting compensatory movement, smoothness and velocity of movement are needed to better inform treatment effects.

Keywords: Stroke, hemiparesis, music therapy, home-care, upper limb rehabilitation, TIMP

\section{Introduction}

Hemiparesis is the most commonly encountered sensorimotor impairment following ischaemic or haemorrhagic stroke (Sabini et al., 2013), occurring in $80 \%$ of patients (Adey-Wakeling \& Crotty, 2013) and with the upper limb being particularly resistant to existing interventions. Research indicates that people with stroke recover significantly more upper limb function if there is less impairment in their upper limb initially caused by the stroke (Coupar et al., 2012). Right hemisphere stroke is reported as being more resistant to upper limb rehabilitation treatment than left (Coupar et al., 2012), possibly due to the predominance of right handedness amongst populations and the left hand being less cortically represented. According to some data (Kwakkel et al., 2004) an increase in treatment dosage in the first six months post stroke improves outcomes and increases recovery rate. Other evidence supports continued recovery of upper limb function at the chronic stage, beyond six months post-stroke (Barrett \& Meschia, 2013; Giaquinto et al., 1994). Movement disorders such as dystonia, tremors and apraxia may occur at stroke onset, sometime after (several years in some cases), or as a progressive 
condition (Handley et al., 2009). Factors such as socioeconomic status (SES), personal circumstances and individual personality traits might influence level of recovery (Adey-Wakeling \& Crotty, 2013; Putman et al., 2007). One study found that motor assessment scores for those who had attained higher educational achievement and income were significantly higher than for those of lower SES, potentially due to those of higher SES being more proactive in seeking coping strategies and problem solving, and thus counteracting their experience of uncertainty with regard to recovery (Putman et al., 2007). How this information would inform or should influence clinical reasoning is uncertain, and is beyond the scope of this article. In summary, there is large variability in the extent of impairment between stroke patients (Langhorne et al., 2011). Using pooled data from 31 reviews, moderate quality evidence was reported for seven stroke upper limb interventions. The review concludes that no high quality evidence for any interventions used within standard care currently exists. This is based on the specific methods of assessing study quality for this Cochrane review, including: risk of bias due to allocation concealment, blinding of participants and data collectors, and selective reporting. The authors state that further RCTs are required with a particular focus on determining treatment dosage (Pollock et al., 2014). In addition, there is limited published research regarding interventions delivered in the home environment for stroke rehabilitation (Fens et al., 2013; Mcintyre et al., 2016), home-based music therapy interventions (Schmid \& Ostermann, 2010), and home-based upper limb interventions for stroke (Turton et al., 2017).

Music interventions for brain-injured populations have been developed, implemented and researched, producing a growing body of evidence for their efficacy (Magee et al., 2017). In recent years, neurologic music therapy (NMT) has presented and, to a degree, formalised a range of music-based interventions (Thaut \& Hoemberg, 
2014). TIMP musical experiences are designed to target specific movement patterns with maximum repetition. Although it is possible and important for patients to identify movements that impair their activities of daily living, it is the music therapist's role to design the music to facilitate the targeted movement patterns. TIMP involves the planning of functional exercises using musical instrument playing to meet functional physical goals, set within the multidisciplinary team, with the aim of transferring the therapeutic learning into real-world applications (Thaut \& Hoemberg, 2014). In addition to playing musical instruments in various spatial configurations to target movement range, TIMP employs external audio cues for the priming and timing of movement and for patients to synchronise their movements in order to improve movement quality (Thaut, 2008; Thaut \& Hoemberg, 2014).

The tactile and auditory feedback from playing instruments as part of structured programs of upper limb rehabilitation following stroke have led to an effect termed as audio-motor coupling. This means that the auditory cortices support motor function (Schneider et al., 2007; Altenmüller et al., 2009; Schneider et al., 2010). Other research also shows neural reorganisation of audio-motor pathways in stroke patients due to the music they generated during instrumental playing (Rojo et al., 2011).

Since there is relatively little existing research to support TIMP for upper limb rehabilitation (Yoo, 2009), and there are few descriptions of its use in clinical settings (Vaudreuil et al., 2018), the aim of presenting these case studies is to help bridge the gap between NMT training, defined clinical protocols and the challenges that present within everyday clinical practice. 


\section{Method}

The two case studies reported here include qualitative and quantitative data from six weeks of twice weekly TIMP intervention. Follow-up data from the post-intervention period together with descriptive data taken from the research diary are included. These multiple sources of data provide information on factors potentially influencing engagement, adherence and outcomes for each participant. This is intended to inform music therapists on patient suitability for TIMP treatment and treatment delivery. The names of the two participants in these case studies have been changed in order to protect their identity. Informed consent was obtained from the participants. Ethical approval was granted by the Essex Research Ethical Committee (reference 13/EE/0400). The study from which the two case studies have been taken is registered with ClinicalTrials.gov, registration number NCT 02310438.

The TIMP protocol was developed for a home-based, pilot randomised controlled trial (RCT) in collaboration with a volunteer stakeholder with stroke hemiparesis, and in consultation with a specialist stroke rehabilitation physiotherapist (Street et al., 2015). The protocol includes targeted motor patterns or movements/movement sequences, with a different facilitating musical pattern for each of these, instruments and equipment required, positioning of instruments, and details of possible variations (Table 1) (Street et al., 2015). Fine motor exercises were performed by participants using touchscreen instruments on iPads, with Garageband ${ }^{\mathrm{TM}}$ and Thumbjam ${ }^{\mathrm{TM}}$ software installed, and using a touchscreen plectrum requiring pinch-grip. Acoustic instruments on stands and some hand percussion were used for proximal, gross movement. iPads were chosen for pinch-grip and fine motor exercises for the following reasons: 1) portability; 2) flexibility for positioning (stand, table surface, lap); 3) rechargability (no power cables); 4) wider range of sounds including guitars and 
keyboards, giving more tailored choices to meet participants' preferences; 5) less resistance involved in moving the touchscreen plectrum/stylus across the screen as compared to an acoustic guitar; 6) greater auditory feedback using amplification through a speaker with adjustable volume, in comparison to using an acoustic guitar which has no touch sensitivity setting; 7) the guitar may be associated with 'being a musician' and be off-putting to those who consider themselves to have no musical ability; and 8) greater harmonic variation, as the guitar would be limited to a single, open-tuned chord unless chord changes were facilitated.

All percussion equipment, stands and mallets were stored and transported in a large, hard-backed luggage case with two wheels and extending handle. The guitar was transported in a hard case. A small shoulder bag was used for the two iPads (also in cases), speaker, two cables and splitter jack (for connecting two iPads to the speaker simultaneously), metronome and earpiece, finger picks, touchscreen plectrum and research log. The iPad stand (microphone boom stand with iPad clamp fitted) was carried separately. In the case of this small RCT, when the community stroke team discussed potential participants with the researcher, they were able to inform on parking, accessibility and available space in home environments. Before being recruited into the study, a home visit was made by the researcher in order to assess each home and plan the sessions to allow enough time to travel from one property to the next, park, transport equipment, gain access, set up and put away all equipment safely.

\section{INSERT TABLE 1 ABOUT HERE}

The criteria for inclusion for the study were: 1) aged $18+$ (no upper age limit), 2) 3-60 months post stroke, 3) had completed statutory community rehabilitation, 4) 
able to lift their affected upper limb onto a table whilst seated and move one or more fingers, and 5) able to consent to treatment. Participants were excluded if currently receiving interventions for upper limb rehabilitation.

The intervention was found to be feasible to deliver in different home environments, with ten participants completing their 12 scheduled sessions and all data collection over 18 weeks. Qualitative analysis of the structured interviews found that 1 ) TIMP was motivating, 2) facilitating music and instruments were supportive of target upper limb movements, and 3) tolerance for TIMP was high and fatigue low. The shared, interactive nature of the exercises was also reported as an important component of the intervention (Street et al., 2017).

Upper limb movements were synchronized with accompanying, strongly pulsed musical patterns played live by the researcher on an acoustic guitar. Equipment set-up time was 15 minutes. Precise periodicity of the pulse, an essential attribute of TIMP (Thaut \& Hoemberg, 2014), was ensured by the use of a metronome beat audible only to the researcher through an earpiece. Each participant was assessed in the first session by the researcher to determine the most appropriate motor patterns and tempo.

The researcher/interventionist had completed neurologic music therapy training, was experienced in delivering the intervention and in working conjointly with physiotherapists and occupational therapists on upper limb rehabilitation. He had also supervised clinicians' NMT work and completed the NMT fellowship training, presenting video clips of clinical work to the primary trainers and receiving feedback on treatment fidelity to published guidelines. Participants were required to play the exercises for 20 - 30 minutes in each session. This was considered long enough to practice targeted movement patterns, but avoid excess fatigue. 


\section{Case Studies}

\section{Participants' medical information}

As can be seen in Table 2, the two participants were varied in age, type of stroke, handedness, period post stroke, and their pre- and post-treatment Action Research Arm Test (ARAT), (Lyle, 1981) and 9 Hole Peg Test (9HPT) (Kellor et al., 1971) scores.

INSERT TABLE 2 AROUND HERE

\section{Case Study 1: Fiona}

Fiona was a 74 year-old female who had a right hemisphere frontoparietal stroke. Her stroke occurred three months prior to joining this study and she had been in acute care for six weeks. She was right handed and prior to her stroke she had been a highly accomplished, self-taught musician. The occupational therapist in the community stroke team explained that she had loss of sensation in her fingers, affecting proprioceptive feedback. The impact of this was that she could not perform ADLs requiring the picking up and manipulating of objects such as cutlery or play her instrument, the recorder.

Fiona's primary objective was to be able to play music again, initially focusing on achieving the note ' $\mathrm{C}$ ' requiring precise left and right hand finger coordination on her recorder. Secondary to this was holding cutlery without dropping it in order to eat.

\section{Home environment}

Fiona lived with her husband in a large, detached house with a driveway for parking and easy groundfloor access. There were no other people living in the property and no pets. As such, there were no concerns regarding sound levels disturbing neighbours and other 
residents, or accessing with equipment. Initial sessons took place in the kitchen, but Fiona then expressed a preference for them to move to a room upstairs, on the first floor, that she used as an art and music space. The room was more cluttered, but still offered ample space to set up and position equipment, with a table available for positioning the iPad, and two wooden kitchen/dining chairs without arms. All exercises upstairs used the iPad and speaker, so there were no issues with carrying the heavier, bulkier percussion stands up the steep set of stairs. Data collection took place in the kitchen, where a table was available for the ARAT and 9HPT.

\section{TIMP exercise selection}

The first five sessions included TIMP 11 (Table 1) which targets grip, release and ulnar deviation wrist movements using a cabasa. Following this, exercises focused exclusively on pinch-grip (holding the touchscreen plectrum) and finger dexterity using TIMP 8, 9 and 10 with all variations and finger combinations. As Fiona was experiencing loss of sensation, feedback from instruments was important (this is linked to the audio-motor coupling discussed earlier), emphasizing the need for an external speaker to the iPad. Exercises predominantly involved using touchscreen instruments and the Bluetooth speaker was always positioned on the table surface with the iPad so that Fiona could feel the resonance of the speaker through the table each time that she played (the built-in iPad speaker did not give adequate sensory feedback). Sometimes there is a delay with Bluetooth speakers between contact with the touchscreen and the sound being heard (Latency). This can be resolved by connecting the speaker using a cable. When using two iPads a splitter and two cables can be employed.

Monitoring progress and adapting the TIMP protocol 
Using the touchscreen guitar on the iPad enabled Fiona to monitor how many of the target chords she could activate before dropping the plectrum. Initially, when Fiona held the touchscreen plectrum (Figure 1) with opposing thumb and index finger, she could only manage to play four of the chords in the eight-chord sequence. However, in the first session this soon increased to her completing the sequence twice without dropping the plectrum or adjusting her grip on it. In the second session she was able to complete three minutes of playing this pattern without dropping the plectrum or repositioning it once. She began to extend finger and thumb combinations in the second session, holding the plectrum using middle finger and thumb, but dropped it frequently. In session six, she was able to use this finger combination and play twice through the sequence without dropping or repositioning the plectrum. Using the smartbass touchscreen instrument, Fiona made rapid progress in improving finger coordination. In the third session, she reported playing four notes on her recorder for the first time since her stroke. Throughout this process, the positioning of the iPad and the musical structures used to facilitate movements remained unchanged. Tempo adjustments could be made, provided that any increases did not compromise movement quality.

\section{INSERT FIGURE 1 ABOUT HERE}

Figure 1. Touchscreen plectrum held over guitar App on tablet (TIMP 6B, 7D \& 9F).

\section{Fiona's development of a new TIMP pattern variation}

In session 10, whilst playing the iPad touchscreen guitar and plectrum exercise (TIMP 9) with her left hand, Fiona placed the plectrum on the table surface and picked it up to play the chord sequence using her right, unaffected, hand. She did this in order to observe the difference in how it felt and looked between her affected and unaffected 
hands. Following this, I suggested that she place the plectrum on the table top once more and pick it up using her affected side, rather than putting it into this hand using her unaffected hand. We continued to play through the pattern alternating left and right hand fingers. In addition, if the plectrum began to slip in her affected left hand fingers, she would reposition it by manipulating her left hand fingers, rather than reaching over to do this using her right hand. As previously stated, this variation in movement sequence was facilitated using the same music played by the therapist. While Fiona placed the plectrum on the table surface to swap hands, the researcher would continue playing the music at the set tempo and she would rejoin and re-synchronise. It was also possible to pause and count-in, starting together with Fiona, depending on how easily she was able to synchronise.

\section{Results}

Fiona made the most significant score increase in the ARAT (Table 3) from pre- to post treatment out of all study participants in the main study, with an increase of 10 points.. Seventeen points is estimated to be the minimal clinically important increase in ARAT score for non-dominant hemiparetic upper limb (Lang et al., 2008). Fiona continued to improve in this area as shown in the follow-up assessments (weeks 9-18, Table 3). She also achieved the largest improvement in the 9HPT. Whereas she was only able to insert a single peg in her pre-treatment assessment, she achieved all pegs in 60 seconds in the final follow-up.

The highest increase in score over the duration of the study for Fiona was in the pinch subcategory (Table 4), with a total increase of seven points in the ARAT score, followed by the grip subscale which increased by a total score of five. Both of these subcategories require greater finger dexterity, picking up and moving smaller objects 
using various finger and thumb combinations, which correspond with a wide range of ADLs such as personal care, food preparation and holding cutlery.

Summary

The majority of sessions focused on fine motor control, with clear progress shown in exercises requiring pinch-grip. Fiona was able to maintain focus on the exercises throughout the treatment period, synchronizing the required movements with the facilitating music despite some minor impairment in sustained attention and procedural memory. There were no signs of anxiety, but a keen determination to regain musical and ADL skills. The TIMP protocol did not require adaptation due to the home location or environment. The home environment was conducive to exercise delivery with regard to parking, access, space and furniture to facilitate spatial arrangement and positioning of equipment, and sound insulation from neighbours.

INSERT TABLES 3 AND 4 ABOUT HERE

\section{Case Study 2: David}

The second participant was a 61-year-old British male, who suffered a right hemisphere stroke, causing multi-focal infarcts (small areas of dead cells in multiple areas in the brain) in the frontal, parietal and occipital lobes. His stroke occurred 14 months prior to joining the study. David presented with left side hemiparesis and tremors in the left lower and upper limb, particularly when trying to extend his upper limb in order to reach, grasp or release an object. Tremors cause rapid oscillations, usually in the hand, whilst performing an action. They have been associated with lesions in the caudate 
nucleus, thalamus, striatum, cerebellum and brain stem, and sub-thalamic, frontal or parietal infarcts (Handley et al., 2009). Tremors render the performance of self-care and food preparation tasks such as shaving and pouring liquids extremely challenging, if not impossible. David's dominant hand was his paretic, left hand. Following his stroke, he was still able to lift his affected upper limb and move his fingers and thumb slightly. In addition to his stroke and heart problems, David also had a serious lung infection, was nil-by-mouth, taking nutrients through a percutaneous endoscopic gastronomy tube (PEG) directly into his stomach.

David presented as a serious person and understandably concerned about his current state of health. He appeared keen to try TIMP, as evident by his immediate response to the study invitation letter. Before beginning treatment David commented that he did not like nor listen to music and that he could not play music in any form, be it singing a melody or tapping along to music in-time.

David was unstable when walking, using a walking aid, and his Berg balance scale score at acute stage was $12 / 56$, increasing four months before beginning music therapy to 28/56. Due to David's medical complications and balance problems when walking, extra care was required when he transferred from his arm chair to a more upright dining chair, which was required to maintain best posture in order to perform TIMP exercises with greatest efficiency. The PEG bag needed to be carried each time David transferred at the beginning and end of each session and for assessments. This was facilitated either by the researcher, the assessor (separate visits to collect data) or David's wife, ensuring that the tube connecting it to his PEG site (on his stomach) was not pulled and that he was not at risk of falling. David's remarks and demeanour throughout the treatment period indicated his feelings of vulnerability, uncertainty and 
pain, as well as his desire to receive effective interventions, and his hope and determination to recover.

\section{Home environment}

David lived with his wife in a detached house with easy groundfloor access and on street parking. There were no other residents or pets present. On one occasion relatives were visiting, including young children, and remained in the openplan lounge/dining area with the television on at low volume throughout the session. A conservatory was available for the sessions which was tidy and clean with no clutter, but too small to facilitate and position equipment. There was a dining table and chairs, one of which David transferred into from his armchair each session. The dining table was used for ARAT and 9HPT data collection.

\section{TIMP exercise selection}

In the first session, pre-recorded music from the iPad was used to facilitate movement so that the researcher could provide hand-over-hand guidance. This is necessary where participants are not yet able to synchronise movements to the music. The prerecorded facilitating music was the researcher playing the guitar at 50bpm and $60 \mathrm{bpm}$ in order to offer some flexibility for patients with different movement frequencies. Facilitating synchronisation to the music is beneficial to patients as it allows them to use the pulse to cue the priming and timing of movements (planning and execution), which David was struggling to achieve. Independent synchronisation of movements indicates that the external auditory cue has been internalised, with potential to strengthen auditory-motor pathways. Sometimes verbal cueing was required from the researcher in order to assist with synchronisation, such as 'get ready, and play, relax your arm', chanted rhythmically in-time to the music. David commented in session two that he could not do it because he had no sense of rhythm and did not know when to 
play. In following sessions, he commented on his performance regularly, communicating with the researcher that he was 'getting into the swing', or 'getting warmed up', which he felt took about ten minutes, and that if he could hit the cymbal it gave him more confidence.

Initially David played the cymbal set at its lowest point on the stand using an adaptive mallet. This type of mallet is heavier than a drum stick, with a thicker handle and a large, round, rubber end approximately the size of a table-tennis ball. The facilitating music was composed to support a single movement that can be played at a slow tempo setting, whilst still providing temporal cues for movement priming and timing (see Figure 2 for transcription). The main objective was to improve elbow extension and this pattern was used in all sessions, gradually extending the distance of the cymbal from David, and using TIMP 1a (fingers; without a mallet) and 1b (regular mallet). We also used this pattern to improve shoulder adduction and flexion, and elbow flexion by positioning the cymbal on his right side above head height. In the fourth session, during TIMP 1 David relaxed his upper limb, fully extended, down by his side following each cymbal hit, making it part of the movement pattern. This was a spontaneous modification of the playing pattern. He commented that it gave more momentum to his playing, producing more volume and improving performance. The researcher observed that this larger upper limb movement facilitated more repeated flexion movements of greater range, and without compensatory movements of the trunk or shoulder.

All TIMP patterns were played in two-minute blocks, monitored using a timer, which allowed the researcher to frequently assess whether David was experiencing fatigue, shoulder pain or any other discomfort, and to adjust the spatial arrangement of equipment. Vigilance towards pain and fatigue was particularly important with David, 
who was still in a vulnerable state of health and very driven and determined to recover even in the face of pain during and due to treatment. In session five, during TIMP 5 (playing bilaterally crossing midline), David's left upper limb extended well to hit the cymbal, whereas initially he was only just clipping the edge of it. The researcher noted that there were fewer tremors than in the previous session and that they reduced in general as each session progressed. This observation corresponded with David's own observation and communication that he felt warmed-up ten minutes into each session.

The majority of TIMP was delivered using live music played by the researcher to a metronome, which was listened to through an earpiece. Metronome settings usually began at $50 \mathrm{bpm}$, which required more shoulder stability than at a faster tempo when extending the elbow, and increased in speed in order to facilitate more elbow extension repetitions with less strain on the shoulder. Metronome settings for finger exercises did not increase as much as for the more proximal upper limb exercises and maintained a setting between 50 and $60 \mathrm{bpm}$. These metronome settings for fine motor exercises were calculated based on David's existing frequency of movement and were informed by the researcher's clinical experience. Retraining impaired finger movement requires a slower frequency as to allow the participants to focus on isolated finger movements, whilst avoiding the build-up of tension in the larger, proximal muscles. Finer motor movement retraining, if undertaken at a frequency that is too high and beyond the participant's current ability, can cause secondary neuromuscular problems, such as dystonia; also reported as a secondary movement disorder in stroke cases (Handley et al., 2009).

INSERT FIGURE 2 ABOUT HERE (musical transcription of TIMP 1)

Figure 2. Transcription of facilitating music for TIMP 1. 
More focused observation of and exercises for thumb movement using TIMP 6 were required with David as he was able to grip and lift, but not combine these movements with upper limb extensions. Using the Garageband touchscreen guitar, this exercise requires participants to rest their fingers on the edge of the iPad and extend their thumb across as many of the virtual guitar strings as possible before then flexing back across the strings to produce a sound (Figure 3). In other sessions, the focus was on upper limb extension and flexion using the cymbal as described earlier.

\section{INSERT FIGURE 3 ABOUT HERE. SHOWING IPAD AND TOUCHSCREEN GUITAR}

Figure 3. Touchscreen guitar exercise for thumb extensions/flexion (TIMP 6).

The researcher adjusted certain musical parameters for TIMP 5 (used in 7/12 sessions), adding much more crescendo, or intensity, to the strumming of each chord. It was noted that this improved David's synchronisation possibly because the musical cues for his priming and timing of movement, including the required muscle force, were more exaggerated and clearer to him.

David began to rotate the cymbal around and position it so that the manufacturer's name that was printed on the surface became his target for hitting, thus 
autonomously fine-tuning the quality and precision of his movements. This action reflects his understanding of the puropose of exercises and motivation to engage in them and invest thought in how to optimise benefit.

Following an episode of playing in session 4 David demonstrated to his wife his improved range of movement, touching his mouth with the palm side of his hand. This movement is the final item of the ARAT gross movement subscale, indicating that he was not only demonstrating increased capacity to perform ADLs, but also possibly linking this to the assessment. His hand began to tremor as he did this, possibly due to the effort required to stabilise the shoulder and the angle of elbow flexion. There were no tremors as he played the cymbal and bongos in synchrony with the music when positioned extreme right above head height, requiring the same movement and elbow flexion as with hand to mouth, but made with repeated rhythmic movements as opposed to being held in a sustained position.

\section{Results}

A small improvement was found in ARAT scores from pre- to post-treatment (Table 3), as David was unable to complete most tasks at all, or unable to do so within the 60 seconds permitted (120 seconds for the 9HPT), including the gross section. Only the water-pouring task, from one glass into another (ARAT task 7), arguably the one most obviously linked to ADLs, was achievable throughout the study and showed a marked improvement in completion timing as shown in Table 5. The normative data for the timing of this task has been reported as 7.9 seconds (Van, 2001).

\section{Summary}

David was highly motivated, self-monitored throughout the study (not only the treatment period) and showed great resilience in coping with his complex, comorbid medical conditions. TIMP 1, supporting upper limb reaching and flexing movements, 
was the most used pattern over the course of treatment. Drumsticks and adaptive mallets were used and towards the final sessions he used his hands in order to maximize range of movement for reaching and grasping. Quantitative outcome scores did not show any significant increases, but task performance time for ARAT task 7, pouring water from one glass to another, did show significant improvement, indicating improvement in movement quality to perform ADLs (Table 5). TIMP treatment over a more extended period would have led to exercises combining grip and release with upper limb extension exercises. Nine hole peg test scores did not show any significant change from pre- to post-treatment or at follow-up.

There were minimal disruptions to sessions due to activity in the home environment. Visits by relatives did not appear to distract David. When his wife arrived home from work during one or two sessions, David took the opportunity to demonstrate to her the increase in movement range he had achieved.

\section{INSERT TABLE 5 ABOUT HERE.}

\section{Discussion}

Severity of stroke and tailored treatment programs

The following discussion makes some comparison between Fiona and David's demographic background and responses, home environment and musical training. Dosage of treatment, monitoring and measuring change is also discussed, and areas of the protocol where flexibility was beneficial. The discussion ends with some advice to clinicians on working conjointly with physiotherapists and occupational therapists when first using TIMP. 
The effects experienced by David following his stroke were clearly more severe than for Fiona. His ARAT baseline score was significantly lower and showed the least post-treatment increase. These factors alone concur with previous research findings, that severity of stroke is a major predictor of upper limb recovery (Coupar et al., 2012). It cannot be ruled out, given her earlier stage post stroke, that Fiona may have been making some spontaneous recovery, which might confound clear identification of treatment effects.

Treatment for both participants was within the context of a RCT, where there was no flexibility for altering the dosage or length of treatment. The question arises as to whether an increased dosage (more weekly sessions) and/or increased length of treatment, $12+$ weeks for example, might have led to greater improvements for the participants, particularly David who had more severe hemiparesis. This is a crucial consideration for music therapists when taking on new referrals and planning treatments, particularly in cases of self-funded treatment or limited allocated funding for rehabilitation services. For example, if a patient is receiving funding for only onceweekly music intervention for six weeks, the clinician being commissioned needs to know whether this is to increase existing dosage of another intervention or as a standalone therapy. If the latter, then this might be insufficient dosage to bring about any change. The clinician should determine whether funds are available for increased weekly sessions over an adequate period of time, provided the patient is deemed fit to tolerate this. Some stroke guidelines state that patients should receive 45 minutes per day of each therapy for as long as they are showing measurable benefit (Intercollegiate Stroke Working Party, 2016). However, research has shown significant recovery of arm function in chronic stroke with three times per-week piano exercises (60 minutes) and bi-weekly self-delivered home practice for three weeks (Villeneuve \& Penhune, 2014). 


\section{Flexibility within protocols}

As shown in these case studies there must be flexibility in such protocols as TIMP. Music therapists have the skills to quickly adapt musical frameworks developed for the synchronisation of clients' specific upper limb movements in order to enhance it. Examples of this are: emphasizing the underlying pulse, building the dynamic contour and intensity towards the beat on which the client plays, mirroring the muscle force required to lift and extend the upper limb. Whilst this can only be achieved through live music delivery, using recorded music in initial sessions enables the music therapist to provide hand-over-hand support to aid clients' movement synchronization. Hand-overhand support can also help with reducing compensatory movements such as extending the trunk in order to aid raising the upper limb. It should be noted, based on the lead author's clinical experience and training, that such hands on support can only be safely and confidently delivered by music therapists who have worked alongside physiotherapists or occupational therapists specializing in upper limb rehabilitation for people with stroke. These clinicians have comprehensive knowledge of anatomy and physiology, and of stroke specific musculoskeletal and neuromuscular complications. When clients reach the point of being able to initiate some variation within an exercise, as Fiona did with the plectrum placed on the table surface and David did with his arm extending between beats by his side, this might indicate that they are familiar enough with the exercise and relaxed enough whilst performing it to be able to think creatively and introduce variations spontaneously in ways that meet their needs.

\section{Home environment}

David and Fiona's home environments did not pose any issues to session delivery with regard to parking, access, sound insulation or space. However, inner city dwellings where parking is unavailable, distanced or costly would require more budgeting, 
planning and time. Accessing apartments with stair or lift access needs consideration when carrying heavy musical equipment. Home-based treatment may also need to be coordinated with daily visits by other health professionals such as physiotherapists and community nurses.

\section{Measuring outcomes and self-monitoring}

Although the ARAT and 9HPT gave an indication of change, these scales do not capture progress in movement quality as in David's case. Changes in movement quality, which includes speed, precision and smoothness of movement, could only be recorded using motion capture systems as shown in related studies (Schneider et al., 2007). Such data would more accurately inform on patient progress, tracking changes that cannot be detected using standard outcome measures, and identifying compensatory movements that could then be reduced, as reported in related literature (Knippenberg et al., 2017). The questions for clinicians are: what assessment tools are needed or will do the job, and who needs to administer them? In some cases both patient and clinician can easily observe that function has changed. The participants described here found the assessment sessions using outcome measures useful for self-monitoring as, even when still unable to complete tasks, they could observe that there were improvements in certain ARAT tasks in terms of reach or timing. From a rehabilitation perspective, speed of task completion does not always correlate with quality of upper limb function as tasks may have been completed with compensatory movements. Again, motion capture technology would more accurately record changes in these movements.

\section{Patient musical training}

Fiona was a highly proficient musician, which may have provided her with more neurological resilience in terms of plasticity, greater bilateral cortical representation of left and right hands, and increased volume of the corpus collosum (Tan et al., 2003; 
Bangert et al., 2006; Grahn \& Rowe, 2009; Wan \& Schlaug, 2010; Lotze et al., 2011). As part of future research into home-based music interventions for upper limb recovery following stroke, it would be useful to collect data on musical training, in order to detect any correlation in the areas of motivation, adherence, tolerance and outcomes, and help inform on treatment dosage in relation to these factors. In contrast, David stated that he did not like music and could not play it, which did not have any observable effect on his sustained high level of motivation to engage in treatment, but may have influenced the potential for neural reorganization within the treatment period and dosage delivered. Progress through the TIMP playing patterns to those requiring more demanding movement sequences may also have been impaired by him not having any previous musical experience in a practical or listening capacity. This appeared to affect his ability to synchronise movements to the facilitating music and benefit from movement timing and quality. Another possibility is that patients will not engage in music therapy at all if they do not like music. In this case, the music therapist could refer the patient to another therapy. However, articulating the benefits of engaging in music-based rehabilitation interventions more clearly might open up otherwise unexplored benefits to patients. People who have received musical training may have more facility for upper limb recovery following stroke, depending on severity, than those who have no musical training, particularly recovery of the non-dominant hand. Musical training has been noted to enhance neural organization (Lotze et al., 2003) and to be associated with greater gray matter volume increases (Levitin, 2013). However, more research is needed specific to stroke recovery and musical training.

\section{Handedness}

In the cases we present, David was affected on his dominant side, meaning that he had more cortical representation of the affected hand due to predominant use. Therefore, 
those dominant motor cortices would be more integrated into other, associated brain regions and neural networks (attention, memory, initiation, executive function). Fiona was required to focus on using her non-dominant left hand. However she had received many years of musical training requiring bilateral hand use, potentially leading to more cortical representation of the non-dominant hand. It is possible that due to a less severe stroke and increased cortical representation of the affected non-dominant hand, Fiona was able to recover more upper limb function with this particular treatment protocol and dosage.

The authors advise music therapists to deliver interventions such as TIMP conjointly with physiotherapists or occupational therapists as often as is possible in order to understand the mechanisms of upper limb hemiparesis and all comorbid sensorimotor impairments. Following this, music therapists will be able to work independently with patients, consulting physiotherapists and other relevant clinicians on specific upper limb issues that may not have been previously encountered. In addition, work of this nature must be viewed from a neurological, top-down perspective, being caused by damage to the brain and nervous system. This is essential in order to maintain focus on how the intervention - the auditory and tactile feedback, motivation and musical interaction - can potentially lead to neural reorganization and improved sensorimotor function. When such change is facilitated it can be observed in improved performance of activities of daily living.

\section{Conclusion}

The case studies presented in this article illustrate that flexibility in the TIMP protocol can support treatment efficacy and adherence in order to achieve the prescribed dosage of target exercises. They also inform on patient suitability based on severity of stroke and time since stroke. In contrast to clinical work, these cases were part of an RCT with 
a prescribed dosage that could not be adjusted. However, the TIMP protocol developed for this study did include variations to meet the variability in participants' upper limb function, such as using adapted musical equipment). 


\section{Acknowledgements}

The authors would like to thank all participants who took part in this research and the community stroke team members who facilitated it.

\section{Disclosure statement}

The authors report no conflicts of interest.

\section{Notes on contributors}

Alexander Street, $\mathrm{PhD}$ is a music therapy practitioner and researcher specializing in the design and delivery of interventions for people with acquired and traumatic brain injury. His work encorporates music technology and interdisciplinary working. Jörg Fachner, DMSc, is Professor for Music, Health and the Brain at Anglia Ruskin University, Cambridge; researching music and consciousness states, depression, stroke and hyperscanning in music therapy.

Wendy L. Magee, $\mathrm{PhD}$, is Professor of Music Therapy at Temple University, Philadelphia specializing in measurement and evidence-based practice in music therapy with people with acquired brain injury. https://orcid.org/0000-0003-4350-1289 


\section{References}

Adey-Wakeling, Z., \& Crotty, M. (2013). Upper limb rehabilitation following stroke: Current evidence and future perspectives. Aging Health, 9, 629+. Retrieved from Academic OneFile; Gale database. Retrieved from http://go.galegroup.com/ps/i.do?id=GALE\%7CA349868051\&v=2.1\&u=anglia itw $\underline{\& i t=r \& p=A O N E \& s w=w \& a s i d=a 257268413 f 83412 \text { da } 7 b 2 b 8 f 9 d 9 f 3199}$

Altenmüller, E., Marco-pallares, J., Münte, T. F., \& Schneider, S. (2009). Neural reorganization underlies improvement in stroke- induced motor dysfunction by music- supported therapy. Annals of the New York Academy of Sciences, 11691(1), 395-405. doi:10.1111/j.1749-6632.2009.04580.x

Baker, F., \& Tamplin, J. (2006). Music therapy methods in neurorehabilitation. London ; Philadelphia: J. Kingsley Publishers. Retrieved from http://site.ebrary.com/lib/anglia/Doc?id=10156048

Bangert, M., Peschel, T., Schlaug, G., Rotte, M., Drescher, D., Hinrichs, H., et al. (2006). Shared networks for auditory and motor processing in professional pianists: Evidence from fMRI conjunction. NeuroImage, 30(3), 917-926. doi:S10538119(05)02415-8 [pii]

Barrett, K. M., \& Meschia, J. F. (2013). Stroke (1st ed.). Hoboken: Wiley

Coupar, F., Pollock, A., Rowe, P., Weir, C., \& Langhorne, P. (2012). Predictors of upper limb recovery after stroke: A systematic review and meta-analysis. Clinical Rehabilitation, 26(4), 291-313. doi:10.1177/0269215511420305 [doi]

Fens, M., Vluggen, T., van Haastregt, J.,C.M., Verbunt, J. A., Beusmans, G. H., \& van Heugten, C.,M. (2013). Multidisciplinary care for stroke patients living in the community: A systematic review. Journal of Rehabilitation Medicine, 45(4), 321. doi:10.2340/16501977-1128

Giaquinto, S., Cobianchi, A., Macera, F., \& Nolfe, G. (1994). EEG recordings in the course of recovery from stroke. Stroke; a Journal of Cerebral Circulation, 25(11), 2204-2209

Grahn, J. A., \& Rowe, J. B. (2009). Feeling the beat: Premotor and striatal interactions in musicians and nonmusicians during beat perception. The Journal of Neuroscience : The Official Journal of the Society for Neuroscience, 29(23), 75407548. doi:10.1523/JNEUROSCI.2018-08.2009 [doi]

Handley, A., Medcalf, P., Hellier, K., \& Dutta, D. (2009). Movement disorders after stroke.(author abstract)(clinical report). Age and Ageing, 38(3), 260

Intercollegiate Stroke Working Party. (2016). National clinical guideline for stroke: Fifth edition (Guidelines No. 5)Royal College of Physicians. (Clinical guideline for stroke) 
Kellor, M., Frost, J., Silberberg, N., Iversen, I., \& Cummings, R. (1971). Hand strength and dexterity. The American Journal of Occupational Therapy : Official Publication of the American Occupational Therapy Association, 25(2), 77

Knippenberg, E., Verbrugghe, J., Lamers, I., Palmaers, S., Timmermans, A., \& Spooren, A. (2017). Markerless motion capture systems as training device in neurological rehabilitation: A systematic review of their use, application, target population and efficacy. Journal of Neuroengineering and Rehabilitation; J.NeuroEng.Rehabil., 14(1) doi:10.1186/s12984-017-0270-x

Kwakkel, C., G., Van Peppen, C., Roland, Wagenaar, C., R., Wood Dauphinee, C., Sharon, Richards, C., C., Ashburn, C., A., et al. (2004). Effects of augmented exercise therapy time after stroke: A meta-analysis. Stroke, 35(11), 2529-2539. doi:10.1161/01.STR.0000143153.76460.7d

Lang, C. E., Edwards, D. F., Birkenmeier, R. L., \& Dromerick, A. W. (2008). Estimating minimal clinically important differences of upper-extremity measures early after stroke. Archives of Physical Medicine and Rehabilitation, 89(9), 16931700. doi:10.1016/j.apmr.2008.02.022

Langhorne, P., Bernhardt, J., \& Kwakkel, G. (2011). Stroke rehabilitation. The Lancet, 377(9778), 1693-1702. doi:10.1016/S0140-6736(11)60325-5

Levitin, D. (2013). Neural correlates of musical behaviors: A brief overview. Music Therapy Perspectives, 31(1), 15-24. doi:10.1093/mtp/31.1.15

Lotze, M., Scheler, G., Tan, H. -. M., Braun, C., \& Birbaumer, N. (2003). The musician's brain: Functional imaging of amateurs and professionals during performance and imagery. NeuroImage, 20(3), 1817-1829.

doi:10.1016/j.neuroimage.2003.07.018

Lyle, R. C. (1981). A performance test for assessment of upper limb function in physical rehabilitation treatment and research. International Journal of Rehabilitation Research.Internationale Zeitschrift Fur Rehabilitationsforschung.Revue Internationale De Recherches De Readaptation, 4(4), 483-492

Magee, W. L., Clark, I., Tamplin, J., \& Bradt, J. (2017). Music interventions for acquired brain injury. The Cochrane Database of Systematic Reviews, 1, CD006787. doi:10.1002/14651858.CD006787.pub3 [doi]

Mcintyre, A., Laura, A., Shannon, J., Amanda, M., Julianne, V., David, U., et al. (2016). Effect of time post stroke onset on ability to make functional gains in individuals accessing a home-based stroke rehabilitation program. Archives of Physical Medicine and Rehabilitation, 97(10), e9-e10.

doi:10.1016/j.apmr.2016.08.026

Pantev, C., \& Herholz, S. C. (2011). Plasticity of the human auditory cortex related to musical training. Neuroscience and Biobehavioral Reviews, 35(10), 2140-2154. doi:10.1016/j.neubiorev.2011.06.010 [doi] 
Pollock, A., Farmer, S. E., Brady, M. C., Langhorne, P., Mead, G. E., Mehrholz, J., et al. (2014). Interventions for improving upper limb function after stroke. The Cochrane Database of Systematic Reviews, 11, CD010820.

doi:10.1002/14651858.CD010820.pub2 [doi]

Putman, K., Wit, L. D., Schoonacker, M., Baert, I., Beyens, H., Brinkmann, N., et al. (2007). Effect of socioeconomic status on functional and motor recovery after stroke: A european multicentre study. Journal of Neurology, Neurosurgery and Psychiatry, 78(6), 593

Rojo, N., Amengual, J., Juncadella, M., Rubio, F., Camara, E., Marco-pallares, J., et al. (2011). Music-supported therapy induces plasticity in the sensorimotor cortex in chronic stroke: A single-case study using multimodal imaging (fMRI-TMS). Brain Injury, 2011, 25; Vol.25(7-8; 7-8), 787; 787-793; 793.

doi:10.3109/02699052.2011.576305

Sabini, R. C., Dijkers, M. P., \& Raghavan, P. (2013). Stroke survivors talk while doing: Development of a therapeutic framework for continued rehabilitation of hand function post stroke. Journal of Hand Therapy: Official Journal of the American Society of Hand Therapists, 26(2), 124-30; quiz 131.

doi:10.1016/j.jht.2012.08.002; 10.1016/j.jht.2012.08.002

Schmid, W., \& Ostermann, T. (2010). Home-based music therapy--a systematic overview of settings and conditions for an innovative service in healthcare. $B M C$ Health Services Research, 10, 291-6963-10-291. doi:10.1186/1472-6963-10-291 [doi]

Schneider, S., Schönle, P., Altenmüller, E., \& Münte, T. (2007). Using musical instruments to improve motor skill recovery following a stroke. Journal of Neurology, 254(10), 1339-1346. doi:10.1007/s00415-006-0523-2

Schneider, S., Altenmüller, E., Münte, T., Rodriguez-Fornells, A., \& Sailer, M. (2010). Music-supported training is more efficient than functional motor training for recovery of fine motor skills in stroke patients. Music Perception, 27(4), 271280. doi:10.1525/mp.2010.27.4.271

Street, A. J. Magee, W. L. Odell-Miller, H. Bateman. A. Fachner, J. C. (2015). Home-based neurologic music therapy for upper limb rehabilitation with stroke patients at community rehabilitation stage - a feasibility study protocol. Frontiers in Human Neuroscience, 9(00480) doi:10.3389/fnhum.2015.00480

Street, A. J., Magee, W. L., Bateman, A., Parker, M., Odell-Miller, H., \& Fachner, J. (2017). Home-based neurologic music therapy for arm hemiparesis following stroke: Results from a pilot, feasibility randomized controlled trial. Clin Rehabil, , 0269215517717060. doi:10.1177/0269215517717060

Thaut, M. H. (2008). Rhythm, music, and the brain: Scientific foundations and clinical applications. New York; London: New York ; London : Routledge 
Thaut, M., \& Hoemberg, V. (2014). Handbook of neurologic music therapy. Oxford: Oxford University Press Oxford

Turton, A., Cunningham, P., van Wijck, F., Smartt, H., Rogers, C., Sackley, C. M., et al. (2017). Home-based reach-to-grasp training for people after stroke is feasible: A pilot randomised controlled trial. Clinical Rehabilitation, 31(7), 891-903. doi:10.1177/0269215516661751

Van, d. L. (2001). The intra- and interrater reliability of the action research arm test: A practical test of upper extremity function in patients with stroke doi:S00039993(01)51301-5 [pii]

Vaudreuil, R., Avila, L., Bradt, J., \& Pasquina, P. (2018). Music therapy applied to complex blast injury in interdisciplinary care: A case report. Disability and Rehabilitation, 1-10. doi:10.1080/09638288.2018.1462412

Villeneuve, M., \& Penhune, V. (2014). A piano training program to improve manual dexterity and upper extremity function in chronic stroke survivors. Frontiers in Human Neuroscience, 8 (2014) doi:10.3389/fnhum.2014.00662

Wan, C. Y., \& Schlaug, G. (2010). Music making as a tool for promoting brain plasticity across the life span. The Neuroscientist : A Review Journal Bringing Neurobiology, Neurology and Psychiatry, 16(5), 566-577.

doi:10.1177/1073858410377805 [doi]

Yoo, J. (2009). The role of therapeutic instrumental music performance in hemiparetic arm rehabilitation. Music Therapy Perspectives, 27(1), 16-24 
Table 1. TIMP playing patterns with equipment, directions for set-up, instructions to patients, and pattern variations.

\begin{tabular}{|c|c|c|c|c|c|c|c|}
\hline & TARGET MOVEMENT ${ }^{1}$ & $\begin{array}{l}\text { INSTRUMENT/S } \\
\& \text { EQUIPMENT }\end{array}$ & POSITIONING & $\begin{array}{l}\text { PARTICIPANT } \\
\text { INSTRUCTIONS } \\
\end{array}$ & $\begin{array}{l}\text { PLAYING } \\
\text { PATTERN }\end{array}$ & $\begin{array}{l}\text { FACILITATING } \\
\text { MUSIC } \\
\end{array}$ & VARIATIONS \\
\hline 1 & $\begin{array}{l}\text { Single beat with affected side: } \\
\text { Elbow and shoulder flexion } \\
\text { \& extension, shoulder } \\
\text { abduction and adduction. } \\
\text { Some wrist extension or } \\
\text { deviation, finger extension, } \\
\text { wrist between pronation and } \\
\text { supination, core muscles. } \\
\text { Note: variation A enables the } \\
\text { above movements in } \\
\text { combination with individual } \\
\text { finger extensions. }\end{array}$ & $\begin{array}{l}14 \text { " Cymbal on } \\
\text { boom stand, } \\
\text { adaptive beater } \\
\text { and wooden } \\
\text { drum stick, or } \\
\text { finger picks } \\
\text { slotted over } \\
\text { thumb and/or } \\
\text { finger/s }\end{array}$ & $\begin{array}{l}\text { Cymbal } \\
\text { positioned at a } \\
\text { distance and } \\
\text { height that the } \\
\text { participant can } \\
\text { reach to play } \\
\text { Gradually raise } \\
\text { height/increase } \\
\text { distance and } \\
\text { angle to } \\
\text { facilitate } \\
\text { increased } \\
\text { movement } \\
\text { range }\end{array}$ & $\begin{array}{l}\text { Relax } \\
\text { shoulders, feet } \\
\text { flat as possible } \\
\text { on floor/foot } \\
\text { rests. Extend } \\
\text { upper limb and } \\
\text { fingers to } \\
\text { cymbal. Relax } \\
\& \text { rest hand on } \\
\text { lap or by side } \\
\text { after playing } \\
\text { Prompt can be } \\
\text { sung to music: } \\
\text { 'reach \& reach } \\
\& \text { play, relax' }\end{array}$ & $\begin{array}{l}\text { Affected side } \\
\text { always play on } \\
\text { beat } 3 \text { of each bar }\end{array}$ & $\begin{array}{l}\text { C, C, Am, } \\
\text { Am, F, G, C, } \\
\text { C } 8 \text { bar chord } \\
\text { sequence in } \\
4 / 4 \\
\text { Arpeggiating } \\
\text { quavers, semi- } \\
\text { quavers, } \\
\text { building the } \\
\text { intensity of } \\
\text { each chord } \\
\text { towards beat } 3 \\
\text { (hit cymbal), } \\
\text { back to semi- } \\
\text { quavers } \\
\text { Each cycle of } \\
\text { the sequence } \\
\text { requires } 8 \\
\text { beats of the } \\
\text { cymbal }\end{array}$ & $\begin{array}{l}\text { A: using } \\
\text { hand/fingers/ } \\
\text { finger picks } \\
\text { B: adaptive } \\
\text { beater or drum } \\
\text { stick } \\
\text { C: alternating } \\
\text { affected \& } \\
\text { unaffected side } \\
\text { (bell of cymbal } \\
\text { on one beat) }\end{array}$ \\
\hline
\end{tabular}


$2 \quad 2$ successive beats with affected side:

\section{Elbow flexion \&}

extension, slight shoulder extension and adduction, wrist between pronation and supination, grip, core muscles

\section{Cymbal on}

boom \& bongos

on stand,

adaptive beater

and $2 \mathrm{X}$ wooden

drum sticks,

finger picks

\section{Bongos on}

affected side, at

achievable height

$\&$ angled so that

larger one is

further from

participant,

cymbal for

unaffected side.

Gradually

reposition for

increased range

of movement
Try to focus

on elbow

bending \&

stretching

Relax

shoulders,

feet flat as

possible on

floor, reach

upper limb

(and

fingers).

Relax upper

limb \& rest

hand on lap

after playing

Sung

prompt: Left

(affected) \&

left \& right,

relax
In $6 / 8$, play on

the underlying

pulse: affected

hand plays

bongo 1, then 2

Rhythmic,

energetic, jazz hand/fingers/

idiom: G7,

G\#Dim, Am7,

D7, played in

6/8 over 4

bars.

Arpeggiated

and/or

strummed

finger picks

with strongly

accented beats

Each cycle of

chords

requires 6

beats on the

percussion (4

on affected

side) (affected) drum

stick

(unaffected)C:

drum stick both

hands
B: adaptive beater 
$3 \quad 3$ successive beats with affected side:

\section{Shoulder and elbow} flexion, \& extension, shoulder abduction, slight shoulder adduction, wrist between pronation and supination, grip, core muscles
Cymbal on boom and bongos on stand, adaptive beater and wooden drum sticks, finger picks
Bongos on affected side (as above), adjusting the height and positioning of instruments to facilitate greater shoulder \& elbow extension or shoulder abduction on elbow bending \& stretching

Sung prompt: Left (affected) \&

left \& left and right
In $6 / 8$, play on underlying pulse: affected side plays bongo 1 , then 2 , then cymbal, then unaffected side plays cymbal bell (hitting the centre of the cymbal)
Any 1,6,2,5 A: using

chord hand/fingers/

sequence

finger picks

played over 4

bars. Strongly B: adaptive beater

pulsed

arpeggios or

strummed

rhythm in $6 / 8$

Each cycle of (affected side) and drum stick (unaffected)

C: two drum sticks

chords

requires 8

beats on the

percussion $(6$

on affected

side)

\begin{tabular}{l}
\hline 4 successive beats with \\
affected side: \\
Shoulder \& elbow flexion \\
\& extension, shoulder \\
abduction, slight shoulder \\
adduction, wrist between \\
pronation and supination, \\
grip, core muscles
\end{tabular}

Try
on
ben
str
Su
pro
TIL

\begin{tabular}{|c|c|c|c|}
\hline $\begin{array}{l}\text { y to focus } \\
\text { elbow } \\
\text { nding \& } \\
\text { etching } \\
\text { ing } \\
\text { ompts as } \\
\text { MP } 2 \& 3\end{array}$ & $\begin{array}{l}\text { In } 6 / 8 \text {, on } \\
\text { underlying } \\
\text { pulse: affected } \\
\text { side plays } \\
\text { bongo } 1 \text {, then } \\
2, \text { then cymbal, } \\
\text { then cymbal } \\
\text { bell, unaffected } \\
\text { side then plays } \\
\text { the pattern in } \\
\text { reverse order }\end{array}$ & $\begin{array}{l}\text { Any } 1,6,2,5 \\
\text { arpeggiated or } \\
\text { strummed } \\
\text { chord } \\
\text { sequence } \\
\text { played over } \\
\text { two bars, } \\
\text { strongly } \\
\text { pulsed rhythm } \\
\text { in } 6 / 8 \\
\text { Each cycle of } \\
\text { chords } \\
\text { requires } 8 \\
\text { beats from the } \\
\text { affected side }\end{array}$ & $\begin{array}{l}\text { A: using } \\
\text { hand/fingers/ } \\
\text { finger picks } \\
\text { B: two drum } \\
\text { sticks, affected } \\
\text { side plays the } \\
\text { pattern, then } \\
\text { unaffected side } \\
\text { plays pattern in } \\
\text { reverse while } \\
\text { affected side rests }\end{array}$ \\
\hline
\end{tabular}




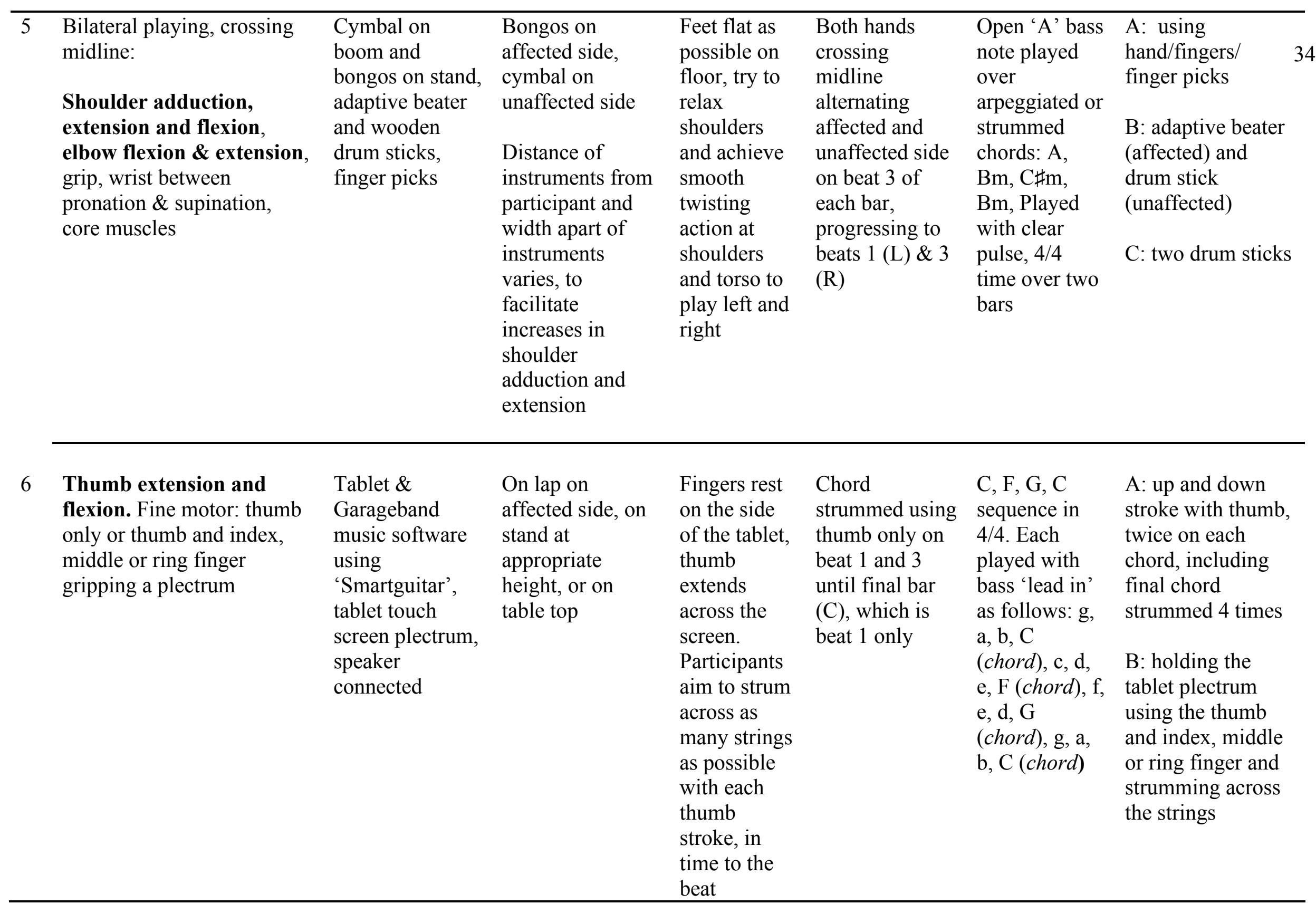


7 Fine motor control of fingers or upper limb: single or two finger combinations using various fingers, or pinch grip

Shoulder stabilisation, finger extension (any/all finger/s or part of finger), wrist deviation, elbow extension, wrist extension

\section{Tablet \&}

Garageband

with

'Smartpiano', sustain switch on, chords set to $G$ (Left side of screen) and $\mathrm{G}$, D5, G in centre of screen, speaker connected.

Alternatively, Smartguitar using the plectrum
In line with

affected side. A

a height

approximate to a

standard

keyboard height,

or that does not

demand

participant to

extend upper

limb excessively

to reach the keys.

In some instances placing the tablet

on the participant's lap

or a table

(affected side)

may offer the

most accessibility

and focus on

finger extension

\section{Relax}

shoulders,

reach with

your

finger/s, feet

flat as

possible on

floor

Participants

may initially

trigger the

sounds using

knuckle or

other part of

finger

The therapist

should

encourage

and support

use of finger

tips where

possible tablet: playing

a single ' $\mathrm{G}$ '

chord by

sliding finger

tip/s, knuckle,

etc, vertically

upwards over

the chord

and/or slightly

away from

body

\section{Alternating}

affected and

unaffected side,

$1^{\text {st }}$ beat of each

bar

(n)

Frere Jacques A: unaffected

in $\mathrm{G}$ major fingers play the $\mathrm{G}, 35$

with strong

pulse

D5, G, chords on

'ding, dang, dong'

lyric section of

song

B: affected side plays $\mathrm{G}, \mathrm{D} 5, \mathrm{G}$ section

C: affected hand uses index, then middle finger to play ' $\mathrm{G}$ ' unaffected hand plays $\mathrm{G}, \mathrm{D} 5, \mathrm{G}$ section

D: using the tablet plectrum and Smartguitar 
$8 \quad$ Fine motor control: single to 4 finger combinations

\section{Finger movements or}

finger extensions, shoulder stabilizing, elbow flexion and extension, shoulder extension, abduction and adduction. Core muscles
2 X tablets: both mounted on a single microphone stand Garageband music software using

'Smartpiano'.

Tablets

connected to speaker using two mini jack leads and a splitter input
Initially, therapist instructs above the other or next to each other, mounted on two tablet participants whilst clamps which are both on the same microphone stand playing the chords by singing the or 'play' each time the participant is required to play the next

chord. chord names

\section{Any finger or}

finger

combination

playing each

chord

individually in

time to the

music. Finger

can extend to

play ascending

chord or flex to

play

descending

chord (down

\section{8 chord}

sequence

played to

accompany

the alternating

chords on

tablet 1 and

tablet 2 as

follows: C, G,

Am, Em, F,

G, C, C. with

each chord

arpeggiated.

Clear accents

on bass note

of each chord

to emphasize

pulse
A: single finger

extension

alternating left and

right hands

B: single finger

extensions,

affected hand only

C: 2 fingers

alternating left and

right hands (index

$\mathrm{L}$, index $\mathrm{R}$, middle

$\mathrm{L}$, middle $\mathrm{R}$, etc)

D: alternating left and right hands using 3 fingers (as above)

E: alternating left and right hands using 4 fingers (as above) 
$9 \quad$ Fine motor control: single to 4 finger combinations or thumb and finger pinch grip using tablet plectrum

Finger movements or finger extensions, Thumb, Index, middle and ring finger extension and flexion, shoulder stabilization, elbow flexion, core muscles
Tablet positioned in portrait rather than landscape in order to facilitate 'keyboard' style finger patterns on the strings.

Tablet on stand, or on

participant's lap and speaker,

stand or resting

on lap or table

top, tablet

plectrum
Relax

shoulders,

feet flat as

possible on

floor

Playing on the

first beat, then

$1 \& 3$, then 1 ,

2,3 .

Initially,

sing each

chord name
Chord

sequence: $\mathrm{C}$.

G. Am, Em,

(one chord per

bar)

C: index, middle
ring

D: index, middle, ring, little finger

E: thumb, index and other fingers

F: using tablet plectrum held using thumb and index, middle or ring finger and used to play individual notes on each string or chords 
10 Fine motor control: single to 2 finger combinations

\section{Finger movements or}

finger extensions, finger, wrist, elbow or shoulder extensions, some shoulder abduction or adduction depending on positioning
Tablet: mounted on the boom stand, speaker connected.

ThumbJam Cello, E3 to E4, major scale setting
Keep finger

in contact

with screen

for the full

duration of

the music,

moving it

up/changing

finger in

time with the

music

Sing 'play'

to

participants

each time

they are

required to

move to the

next note

11 Grip, wrist

\section{ulnar and radial}

deviation, elbow flexion

(Baker \& Tamplin, 2006)

Cabasa: small,
medium and
large sizes

Bongos or

cymbal on stand

\begin{tabular}{|c|c|}
\hline $\begin{array}{l}\text { Cabasa held in } \\
\text { unaffected hand, } \\
\text { affected hand } \\
\text { aims to grip the } \\
\text { beads over the } \\
\text { top/round the } \\
\text { side with any } \\
\text { finger/thumb } \\
\text { combination and }\end{array}$ & $\begin{array}{l}\text { Relax } \\
\text { shoulders, } \\
\text { 'twist, } \\
\text { release, } \\
\text { reach, play' } \\
\text { (spoken or } \\
\text { sung to } \\
\text { participants }\end{array}$ \\
\hline
\end{tabular}

Playing up the screen: E, F\#, $\mathrm{G} \#, \mathrm{~A}, \mathrm{~B}, \mathrm{C} \#$,

$\mathrm{D} \#, \mathrm{E}$
Ascending

scale: E, B, E, sustaining the top 38

A, E, A, B, E. ' $E$ ' note of the

Descending scale at the top of

scale: E, B, A, the screen, then

E, A, E, B, E . following the

Arpeggiating music to descend

the chords and back down the

with a 'turn

around' screen to bottom

phrase at the

end to

indicate 'back

to the start

note (E)' For

example the

notes: b, c\#,

$\mathrm{d} \#$ on the ' $\mathrm{B}$ '

string

$\begin{array}{lll}\begin{array}{l}\text { Playing on 1 } \\ \text { (cabasa twist) }^{\text {st }}\end{array} & \begin{array}{l}\text { Strongly } \\ \text { pulsed, }\end{array} & \begin{array}{l}\text { A: grip and twist } \\ \text { cabasa with }\end{array} \\ {\text { and } 3^{\text {rd }}}^{\text {(bongo/cymbal) }} & \text { rhythmic and } & \text { affected hand on } \\ \text { beat of each bar } & \text { music, for } & \text { beat 1, then release } \\ & \text { it and hit the } \\ & \text { Spample } & \text { cymbal on beat 3 } \\ & \text { using E, F/E } & \text { using the affected } \\ & \text { bass, 4/4 time } & \text { beater) }\end{array}$




\begin{tabular}{|c|c|c|c|c|c|c|c|}
\hline 12 & $\begin{array}{l}\text { Wrist and foreupper limb } \\
\text { pronation \& supination, } \\
\text { shoulder adduction, elbow } \\
\text { extension, core muscles }\end{array}$ & $\begin{array}{l}\text { Cymbal on } \\
\text { boom \& bongos } \\
\text { on stand, two } \\
\text { drum sticks or } \\
\text { beaters taped } \\
\text { together so there } \\
\text { is a tip at either } \\
\text { end or single } \\
\text { stick/beater } \\
\text { rotated so that } \\
\text { tip plays cymbal } \\
\text { then bongo }\end{array}$ & $\begin{array}{l}\text { Cymbal and } \\
\text { bongos slightly } \\
\text { less than a } \\
\text { beater's distance } \\
\text { apart focusing } \\
\text { efforts on wrist } \\
\text { and foreupper } \\
\text { limb rotation } \\
\text { Alternatively, } \\
\text { play bongos, } \\
\text { rotating } \\
\text { wrist/foreupper } \\
\text { limb to play } \\
\text { bongo 1 then } \\
\text { bongo 2 }\end{array}$ & $\begin{array}{l}\text { Sung } \\
\text { prompt: turn } \\
\text { \&turn \& } \\
\text { play }\end{array}$ & $\begin{array}{l}\text { Affected hand } \\
\text { only plays } \\
\text { bongo with one } \\
\text { end of the stick, } \\
\text { then cymbal or } \\
\text { bongo } 2 \text { with } \\
\text { the other end } \\
\text { on beat } 3 \text { only } \\
\text { (slow rotation) } \\
\text { or } 1 \& 3\end{array}$ & $\begin{array}{l}\text { Octaves or } \\
\text { chords: F } \\
\text { slide to } \mathrm{C}, \\
\text { slide to } \mathrm{Gm}, \\
\text { slide to } \mathrm{Dm} \\
4 / 4 \text { time over } \\
2 \text { bars, with } \\
\text { strong accent } \\
\text { on beats } 1 \& 3 \\
\& \text { crescendo } \\
\text { between each } \\
\text { chord }\end{array}$ & $\begin{array}{l}\text { A: holding just the } \\
\text { adaptive beater } \\
\text { and making full } \\
\text { foreupper limb } \\
\text { pronation and } \\
\text { supination } \\
\text { movement to play } \\
\text { cymbal then bongo } \\
\text { or bongo } 1 \text {, bongo } \\
2 \\
\text { B: using a cabasa } \\
\text { instead of } \\
\text { stick/beater }\end{array}$ \\
\hline
\end{tabular}

Note. 1. Under 'Target Movement' the associated muscle groups are written in bold type. 
Table 2. Demographic and clinical data

\begin{tabular}{lllllllll}
\hline & Gender/Age & $\begin{array}{l}\text { Stroke } \\
\text { Type }\end{array}$ & $\begin{array}{l}\text { Side } \\
\text { affected }\end{array}$ & $\begin{array}{l}\text { Months } \\
\text { post } \\
\text { stroke }\end{array}$ & $\begin{array}{l}\text { ARAT }^{2} \\
\text { Pre- }\end{array}$ & $\begin{array}{l}\text { ARAT } \\
\text { Post- }\end{array}$ & $\begin{array}{l}\text { 9HPT } \\
\text { Pre- }\end{array}$ & $\begin{array}{l}\text { 9HPT } \\
\text { Post- }\end{array}$ \\
\hline Fiona & $\mathrm{F} / 74$ & Embolic & $\begin{array}{l}\text { Left-non- } \\
\text { dominant }\end{array}$ & $\begin{array}{l}3 \\
\text { months }\end{array}$ & $35 / 57$ & +10 & $1 / 120$ & $15 / 12 \mathrm{~s}$ \\
David & $\mathrm{M} / 61$ & Ischaemic & $\begin{array}{l}\text { Left- } \\
\text { dominant }\end{array}$ & $\begin{array}{l}14 \\
\text { months }\end{array}$ & $12 / 57$ & +1 & $1 / 120 \mathrm{~s}$ & $1 / 120 \mathrm{~s}$ \\
\hline
\end{tabular}

Note. 2. Action Research Arm Test. 3. Nine Hole Peg Test. 
Table 3. ARAT and 9HPT scores for Fiona and David

\begin{tabular}{|c|c|c|c|c|}
\hline & \multicolumn{2}{|c|}{ Fiona } & \multicolumn{2}{|c|}{ David } \\
\hline & $\begin{array}{l}\text { ARAT score } \\
\text { of } 57\end{array}$ & $\begin{array}{l}\text { 9HPT } \\
\text { score }\end{array}$ & $\begin{array}{l}\text { ARAT score } \\
\text { of } 57\end{array}$ & $\begin{array}{l}\text { 9HPT } \\
\text { scores }\end{array}$ \\
\hline $\begin{array}{l}\text { Week } 1 \\
\text { Pre- } \\
\text { treatment }\end{array}$ & 35 & $\begin{array}{l}1 \text { in } 120 \\
\text { seconds }\end{array}$ & 12 & $\begin{array}{l}1 \text { in } 120 \\
\text { seconds }\end{array}$ \\
\hline $\begin{array}{l}\text { Week } 6 \\
\text { Post- } \\
\text { treatment }\end{array}$ & 45 & $\begin{array}{l}15 \text { in } 120 \\
\text { seconds }\end{array}$ & 13 & $\begin{array}{l}1 \text { in } \\
120 \text { seconds }\end{array}$ \\
\hline $\begin{array}{l}\text { Week } 9 \\
\text { Washout }\end{array}$ & 49 & $\begin{array}{l}\text { All in } 75 \\
\text { seconds }\end{array}$ & 17 & $\begin{array}{l}2 \text { in } 120 \\
\text { seconds }\end{array}$ \\
\hline $\begin{array}{l}\text { Week } 15 \\
\text { Follow-up }\end{array}$ & 48 & $\begin{array}{l}\text { All in } 77 \\
\text { seconds }\end{array}$ & 16 & $\begin{array}{l}0 \text { in } 120 \\
\text { seconds }\end{array}$ \\
\hline $\begin{array}{l}\text { Week } 18 \\
\text { Follow-up }\end{array}$ & 50 & $\begin{array}{l}\text { All in } 60 \\
\text { seconds }\end{array}$ & 16 & $\begin{array}{l}4 \text { in } 120 \\
\text { seconds }\end{array}$ \\
\hline
\end{tabular}


Table 4 . ARAT subscale scores for Fiona

\begin{tabular}{lllllll}
\hline $\begin{array}{l}\text { ARAT } \\
\text { subscale }\end{array}$ & Week 1 & Week 6 & Week 9 & Week 15 & Week 18 & $\begin{array}{l}\text { Change } \\
(+/-)\end{array}$ \\
\hline Grasp (/18) & 17 & 18 & 18 & 18 & 18 & +1 \\
Grip (/12) & 7 & 11 & 12 & 12 & 12 & $+5^{*}$ \\
Pinch (/18) & 4 & 7 & 10 & 9 & 11 & $+7^{*}$ \\
Gross (/9) & 7 & 9 & 9 & 9 & 9 & +2 \\
\hline
\end{tabular}


Table 5. David: Changes in time taken to perform ARAT task 7, water pouring.

\begin{tabular}{ll}
\hline ARAT Time-point & Time to complete ARAT task 7 (seconds) \\
\hline Week 1 pre treatment & 44 \\
Week 6 post treatment & 13.16 \\
Week 9 washout & 15.78 \\
Week 15 follow up & 24.73 \\
Week 18 follow up & 19.08 \\
\hline
\end{tabular}


